\title{
Dietary Heat-Killed Lactobacillus brevis SBC8803 Attenuates Chronic Sleep Disorders Induced by Psychophysiological Stress in Mice
}

\author{
Sayaka Higo-YAMAMOTO$^{1}$, Saori YAMAMOTO${ }^{1}$, Koyomi MIYAZAKI $^{1}$, \\ Yasukazu NAKAKITA ${ }^{2}$, Hirotaka KANEDA ${ }^{3}$, Yoshihiro TAKATA ${ }^{3}$, \\ Takeshi NAKAMURA ${ }^{3}$ and Katsutaka OISHI ${ }^{1,4,5,6, *}$ \\ ${ }^{1}$ Biological Clock Research Group, Biomedical Research Institute, National Institute of Advanced \\ Industrial Science and Technology (AIST), Tsukuba, Ibaraki 305-8566, Japan \\ ${ }^{2}$ Frontier Laboratories of Value Creation, Sapporo Holdings Ltd., Yaizu, Shizuoka 425-0013, Japan \\ ${ }^{3}$ Group RED Strategy Department, Sapporo Holdings Ltd., Shibuya-ku, Tokyo 150-8522, Japan \\ ${ }^{4}$ Department of Applied Biological Science, Graduate School of Science and Technology, \\ Tokyo University of Science, Noda, Chiba 278-8510, Japan \\ ${ }^{5}$ Department of Computational Biology and Medical Sciences, Graduate School of Frontier Sciences, \\ The University of Tokyo, Kashiwa, Chiba 277-0882, Japan. \\ ${ }^{6}$ School of Integrative and Global Majors (SIGMA), University of Tsukuba, \\ Tsukuba, Ibaraki 305-8577, Japan
}

(Received November 7, 2018)

\begin{abstract}
Summary We previously reported that dietary heat-killed Lactobacillus brevis SBC8803 affects sleep in mice and humans. The present study examined whether SBC8803 improves psychophysiological stress-induced chronic sleep disorders (CSD) using a mouse model characterized by disrupted circadian rhythms of wheel-running activity and sleep-wake cycles. Mice were fed with a diet supplemented with $0.5 \%$ heat-killed SBC8803 for 6 wk and imposed stress-induced CSD for last 2 wk. Dietary SBC8803 suppressed the reduction in wheel-running activity induced by CSD. Electroencephalography (EEG) revealed that SBC8803 significantly restored wakefulness and increased non-rapid eye movement (NREM) sleep during the second half of the active phase during CSD. The CSD-induced reduction in EEG slow wave activity, a marker of NREM sleep intensity, during the beginning of the inactive phase was significantly improved by SBC8803 supplementation. These findings suggest that dietary heat-killed SBC8803 confers beneficial effects on insomnia and circadian sleep disorders induced by psychophysiological stress.
\end{abstract}

Key Words Lactobacillus brevis, sleep, stress, wheel-running activity, circadian rhythm

Recognition of the importance of sleep as a social, as well as a health problem has brought about worldwide growing interest in sleep and sleep research. Chronic sleep disorders (CSD) are associated with significant and cumulative neurobehavioral deficits and physiological changes that result in hypertension, obesity, diabetes, cardiovascular morbidity, and stroke in addition to disorders such as depression (1). Sleep disorders confer a major burden on society due to being highly prevalent and associated with socio-economic costs including absenteeism and increased rates of accidents in the workplace (2). Mental stress is an important risk factor that contributes to the mechanisms of underlying sleep disorders in humans (3).

Probiotics are usually defined as viable non-pathogenic microorganisms that benefit host health when ingested. Recent neurobiological insights have revealed that the gut microbiota plays important roles in gutbrain crosstalk and is linked to neuropsychological

\footnotetext{
*To whom correspondence should be addressed.

E-mail: k-ooishi@aist.go.jp
}

modulation. Lactobacillus species are widely consumed as probiotic supplements and to prevent acute infectious diarrhea (4) and treat irritable bowel syndrome (5). Dietary heat-killed Lactobacillus brevis SBC8803, a strain isolated from barley, can alleviate allergies (6), improve the intestinal tract barrier (7) and prevent alcoholic liver disease $(8)$ in experimental animals. Human trials have also demonstrated that dietary SBC8803 supplementation improves bowel movements (ameliorating both constipation and diarrhea), liver function and blood triglyceride levels (9). We previously treated healthy mice with SBC 8803 for $4 \mathrm{wk}$ and found that the mice spent significantly less time in non-rapid eye movement (NREM) sleep and spent more time being awake during the latter half of the nighttime (active phase) (10). We conducted a non-randomized, placebo-controlled, double blind, crossover pilot study of volunteers with slightly high scores on the Athens Insomnia Scale (AIS) to determine the effect of SBC8803 consumption on sleep (11). Sleep journals revealed improved wakefulness when SBC8803 was consumed and a marginal effect on drowsiness during the following day. 
Classical mouse models of CSD such as disk-overwater, gentle handling, platform (also called flower-pot), and slowly rotating wheels artificially affect circadian behavioral rhythms by providing circadian time cues, because they are based on daily forced physical sleep deprivation (12). We developed a model of CSD induced by psychophysiological stress characterized by a reduced amplitude of circadian rhythms such as wheel-running, sleep-wake cycles, sleep fragmentation and hyperphagia for several weeks without adaptation (13-15). Plasma levels of catecholamines such as adrenaline and noradrenaline were significantly elevated, whereas corticosterone levels were slightly affected in mice with CSD, suggesting that the stress applied herein activated the sympathetic-adrenal medullary axis (13). The mice with CSD notably had hyperphasia accompanied by hypoleptinemia and impaired glucose tolerance (14, 15), which resembles the situation found in insomniac humans. These model mice would be useful for evaluating the effects of probiotics on stress-induced circadian sleep disorders. The present study aimed to determine whether SBC8803 improves CSD.

\section{MATERIALS AND METHODS}

Preparation of diet containing probiotic bacteria. Lactobacillus brevis SBC8803 $(6,16)$ obtained from Frontier Laboratories of Value Creation, Sapporo Holdings Ltd. (Shizuoka, Japan) was propagated for $24 \mathrm{~h}$ at $30^{\circ} \mathrm{C}$ in broth containing $2 \%$ maltose, $1.4 \%$ yeast extract, $0.5 \%$ sodium acetate, and $0.005 \% \mathrm{MnSO}_{4} \cdot 5 \mathrm{H}_{2} \mathrm{O}$. Bacteria were pelleted by centrifugation at $8,000 \times g$ for $10 \mathrm{~min}$ at $10^{\circ} \mathrm{C}$ (Himac CR21G; Hitachi Koki Co. Ltd., Japan), washed three times with deionized water, heat-killed at $105^{\circ} \mathrm{C}$ for $10 \mathrm{~min}$ and frozen at $-50^{\circ} \mathrm{C}$. The frozen bacteria were lyophilized by drying at temperatures $<30^{\circ} \mathrm{C}$ for $73.3 \mathrm{~h}$, then milled and homogenized through a $60 \mathrm{G}$ mesh filter. The bacterial powder was thoroughly mixed with AIN-93M to a final ratio of $0.5 \%(6,10)$, then the mixture was pelletized at temperatures between $40-50^{\circ} \mathrm{C}$ and dried at $75^{\circ} \mathrm{C}$ for $1-1.5 \mathrm{~h}$ until the water content decreased to $<10 \%$.

Animals. Five-week-old male $\mathrm{C} 3 \mathrm{H} / \mathrm{HeN}$ mice (Japan SLC, Inc., Hamamatsu, Japan) that were individually maintained in plastic cages (length $\times$ width $\times$ height: $34 \times 22.5 \times 15.5 \mathrm{~cm})$ containing animal paper bedding and running wheels (SW-15; Melquest, Toyama, Japan) were fed with a pelletized normal diet (CE-2; CLEA Japan, Inc., Tokyo, Japan) ad libitum under 12-h light-12-h dark cycles (LD 12 : 12; lights on at Zeitgeber time (ZT) 0 and lights off at ZT12) for $1 \mathrm{wk}$. The mice were then fed with purified AIN-93M rodent diet (Charles-River Co. Ltd., Wilmington, MA, USA) with or without (control) $0.5 \%$ heat-killed SBC8803 $(6,16)$ for $6 \mathrm{wk}$. They were then exposed to $2 \mathrm{wk}$ of psychophysiological stress for CSD induction as described (13-15). Briefly, paper-chip bedding was replaced with water to a depth of $1.5 \mathrm{~cm}$ depth which caused the mice to stay on a wheel for $24 \mathrm{~h}$ every day.

Wheel-running activity was continuously recorded at 1-min intervals using Chronobiology Kits (Stanford
Software Systems, Stanford, CA, USA). Food intake during the study was measured and the mice were weighed weekly.

All animal experiments proceeded according to the guidelines for animal experiments published by the National Institute of Advanced Industrial Science and Technology (AIST). The AIST Animal Care and Use Committee approved all experimental protocols (Permissions: \#2015-020 and \#2015-056).

Sleep recording and analysis. Two electroencephalographic (EEG) electrodes were implanted into the mouse skull and fixed with dental cement. Two stainless steel wires were then implanted in the neck muscles to collect electromyographic (EMG) signals. We recorded sleep using a TL11M2-F20-EET telemetric device (Data Sciences International, St. Paul, MN, USA) that was subcutaneously implanted into the backs of the mice according to the manufacturer's instructions under anesthesia with $0.3 \mathrm{mg} / \mathrm{kg}$ i.p. medetomidine, $4.0 \mathrm{mg} / \mathrm{kg}$ i.p. midazolam and $5.0 \mathrm{mg} / \mathrm{kg}$ i.p. butorphanol. After recovering from this procedure for $2 \mathrm{wk}$, polygraphic EEG and EMG traces were continuously recorded from $3 \mathrm{~d}$ immediately before, until the end of the 2 wk of CSD.

Cortical EEG and EMG signals were digitized at a sampling rate of $500 \mathrm{~Hz}$ and recorded using Dataquest A.R.T. ${ }^{\text {TM }}$ (Data Sciences International). We used SLEEPSIGN (Kissei Comtec, Nagano, Japan) to automatically score polygraphic traces offline in 10-s epochs divided into stages of being awake and having rapid eye movement (REM) and non-rapid eye movement (NREM) sleep according to the standard criteria described below. Wakefulness was characterized by low-amplitude EEG signals with mixed frequency components and relatively high, often irregular, EMG activity. The amplitude of EEG activity during NREM sleep was high and dominated by slow-frequency waves of 1.0-4.0 Hz, whereas EMG activity was low. Low-amplitude EEG signals dominated by theta waves of 6.0-9.0 Hz and low EMG activity characterized REM sleep. Defined sleep-wake stages were visually examined and corrected if necessary. Power spectrum density was calculated at $\sim 0.48 \mathrm{~Hz}$ intervals, then the EEG delta and theta frequency bands were set at $0.5-4.9 \mathrm{~Hz}$ and 5.4-7.8 Hz, respectively.

Statistical analysis. All values are expressed as means \pm standard error of the means (SE) and were statistically evaluated using two-way analysis of variance (ANOVA) followed by Tukey's test using Excel-Toukei 2010 software (Social Survey Research Information Co. Ltd., Tokyo, Japan). Statistical significance was established at $p<0.05$.

\section{RESULTS}

Body weight and daily food consumption were identical between the control and SBC8803 mice after $6 \mathrm{wk}$ of SBC8803 supplementation (Supplemental Online Material, Fig. S1). SBC8803 supplementation did not affect reductions in body weight and increases in food consumption induced by CSD.

We compared the diurnal rhythms of running wheel activity between control and SBC8803 mice under nor- 


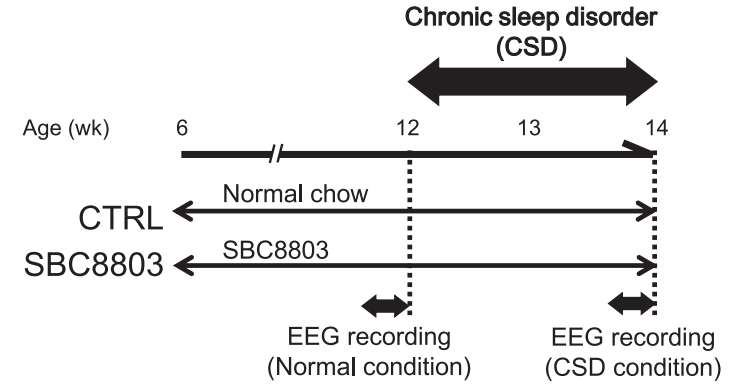

Fig. 1. Experimental design. Six-week-old mice were individually housed in cages with running-wheels and fed ad libitum with AIN-93M without (control) or supplemented with $0.5 \%$ heat-killed SBC 8803 for $6 \mathrm{wk}$ under a 12-h light-12-h dark cycle. The mice were then exposed to psychophysiological stress to induce CSD for 2 wk. Polygraphic electroencephalogram (EEG) and electromyogram (EMG) traces were continuously recorded under normal and CSD conditions. CTRL, control.

A
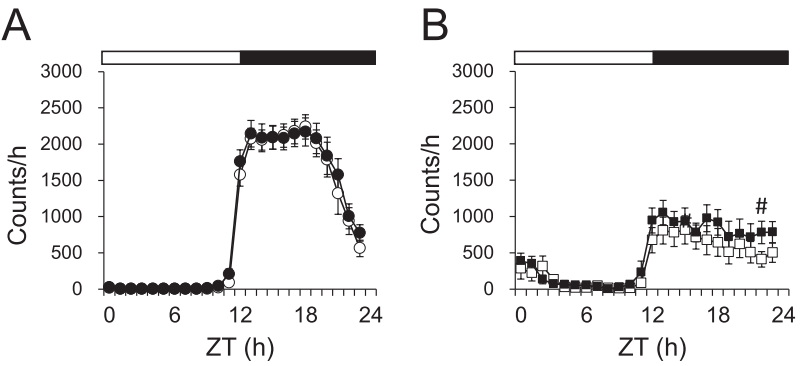

C

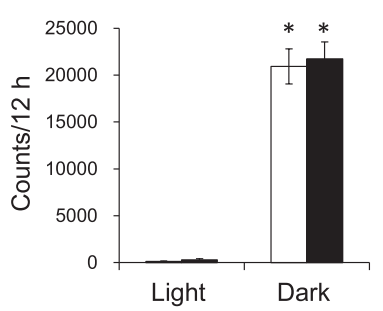

D

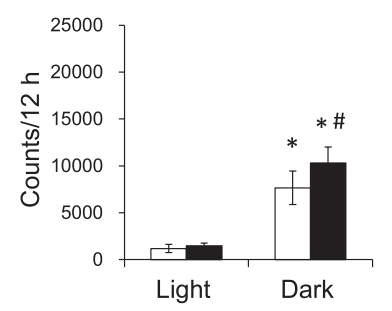

Fig. 2. Dietary heat-killed SBC8803 attenuated the CSD-induced reduction in wheel-running activity during the active phase. Hourly values for wheel running by control (unfilled circles) and SBC8803 (filled circles) mice under normal (A) and CSD (B) conditions. Day-night fluctuation of wheel-running activity by mice fed with (filled bars) or without (unfilled bars) SBC8803 under normal (C) and CSD (D) conditions. Data are shown as means \pm SE $(n=11)$. Significant differences between light and dark phases in each group $\left({ }^{*} p<0.01\right)$, and between control and SBC8803 mice at each time point or under each condition $\left({ }^{\#} p<0.05\right)$. Horizontal unfilled and filled bars indicate light and dark periods, respectively.

mal and CSD conditions (Fig. 1). Six weeks of SBC8803 supplementation did not affect the circadian running wheel activity (Fig. 2A, C). Two weeks of CSD severely dumped the day-night activity rhythm by reducing the nighttime wheel running activity as previously described (12-14). The CSD-induced reduction of nighttime run-
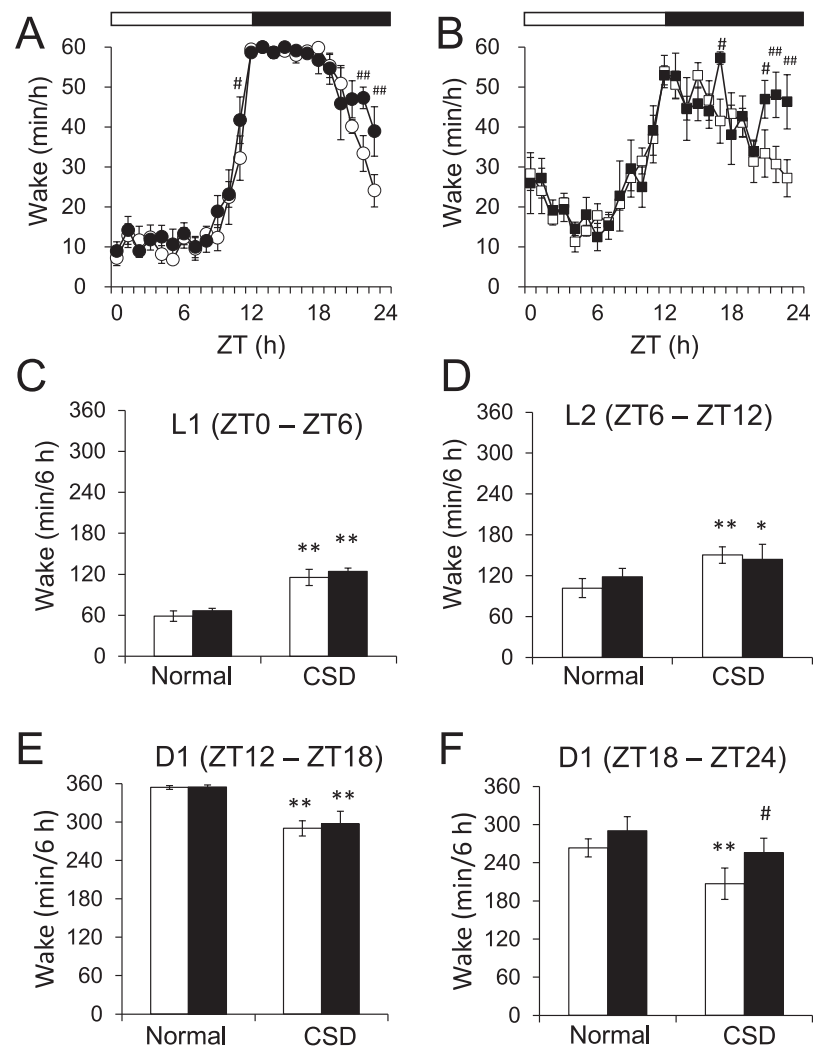

Fig. 3. Dietary heat-killed SBC8803 suppressed the CSD-induced reduction of wakefulness during active phase. Diurnal changes of wakefulness among mice fed with (filled circles) or without (unfilled circles) SBC8803 under normal (A) and CSD (B) conditions. $\mathrm{C}-\mathrm{F}$ : Total time spent in wakefulness every $6 \mathrm{~h}$ by mice fed with (filled bar) or without (unfilled bar) SBC8803 under normal and CSD conditions. C, ZTO-6 (L1); D, ZT6-12 (L2); E, ZT12-18 (D1); F, ZT18-24 (D2). Data are shown as means \pm SE (Control, $n=5$; SBC8803, $n=4$ ). Significant differences between normal and CSD conditions in each group $\left({ }^{*} p<0.05\right.$ and $\left.{ }^{* *} p<0.01\right)$ and between control and SBC8803 mice at each time point or under each condition $\left({ }^{\#} p<0.05\right.$ and $\left.{ }^{\# \#} p<0.01\right)$. Horizontal unfilled and filled bars indicate light and dark periods, respectively.

ning wheel activity was significantly suppressed by SBC8803 supplementation (Fig. 2D).

We used EEG to assess sleep-wake rhythms in mice fed with dietary heat-killed SBC8803. Temporal profiles of sleep-wake rhythms were severely affected by CSD. The duration of wakefulness was significantly increased during the light period (Fig. 3C, D) and decreased during the first half of the dark period (Fig. 3E), in both control and SBC 8803 mice. The duration of wakefulness during the second half of the dark period was also significantly decreased by CSD in control, but not in SBC8803 mice (Fig. 3F). In contrast, the duration of NREM sleep was significantly decreased during the light period (Fig. 4C) and increased during the first half of the dark period (Fig. 4E), in both control and SBC8803 mice. The duration of NREM sleep during the second half of the dark period was significantly increased by CSD in control, but 

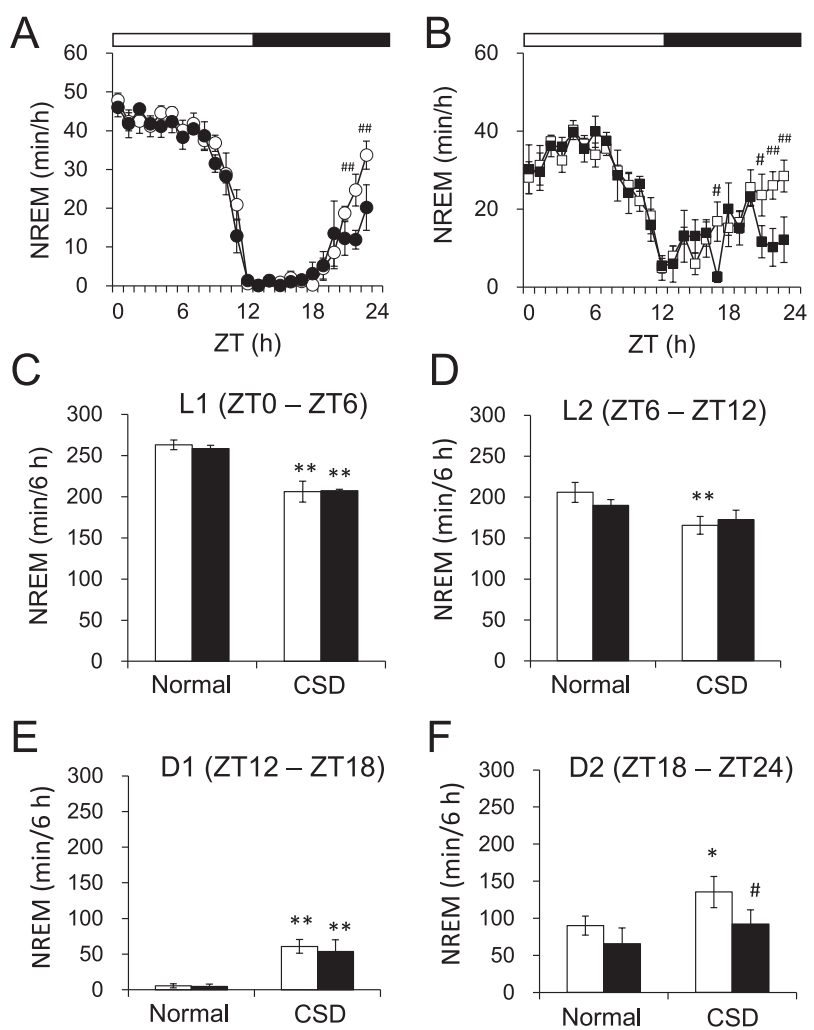

Fig. 4. Dietary heat-killed SBC8803 suppressed the CSD-induced increase in NREM sleep during active phase. Diurnal changes of NREM sleep among mice fed with (filled circles) or without (unfilled circles) SBC8803 under normal (A) and CSD (B) conditions. C-F: Total time spent in NREM sleep every $6 \mathrm{~h}$ before and after CSD by mice fed with (filled bars) or without (unfilled bars) SBC8803 under normal and CSD conditions. C, ZT0-6 (L1); D, ZT6-12 (L2); E, ZT12-18 (D1); F, ZT1824 (D2). Data are shown as means \pm SE (Control, $n=5$; SBC8803, $n=4)$. Significant differences between normal and CSD conditions in each group $\left(^{*} p<0.05\right.$ and $* * p<0.01)$ and between control and SBC8803 mice at each time point or under each condition $\left({ }^{\#} p<0.05\right.$ and $\# p<0.01$ ). Horizontal unfilled and filled bars indicate light and dark periods, respectively.

not in SBC8803 mice (Fig. 4F). The duration of REM sleep was significantly increased throughout the dark period in both control and SBC8803 mice (Fig. 5E, F). The daily total amount of time spent in wakefulness, NREM sleep, and REM sleep did not statistically differ between control and SBC8803 mice regardless of CSD (Supplementa1 Online Material, Fig. S2).

Figure 6A shows a significant decrease in EEG delta/ theta ratio (SWA) in NREM sleep during the first half of the light period under CSD in both control and SBC8803 mice. The average EEG delta/theta ratio during ZT4-6 was significantly decreased by CSD in control, but not in SBC8803 mice (Fig. 6B). We then determined the EEG power density spectra of NREM sleep at ZT4 under normal (Fig. 7A) and CSD (Fig. 7B) conditions. The power spectrum analysis of NREM sleep revealed significantly increased delta and significantly reduced theta activities at ZT4 in the SBC8803 mice (Fig. 7B-D). Dietary
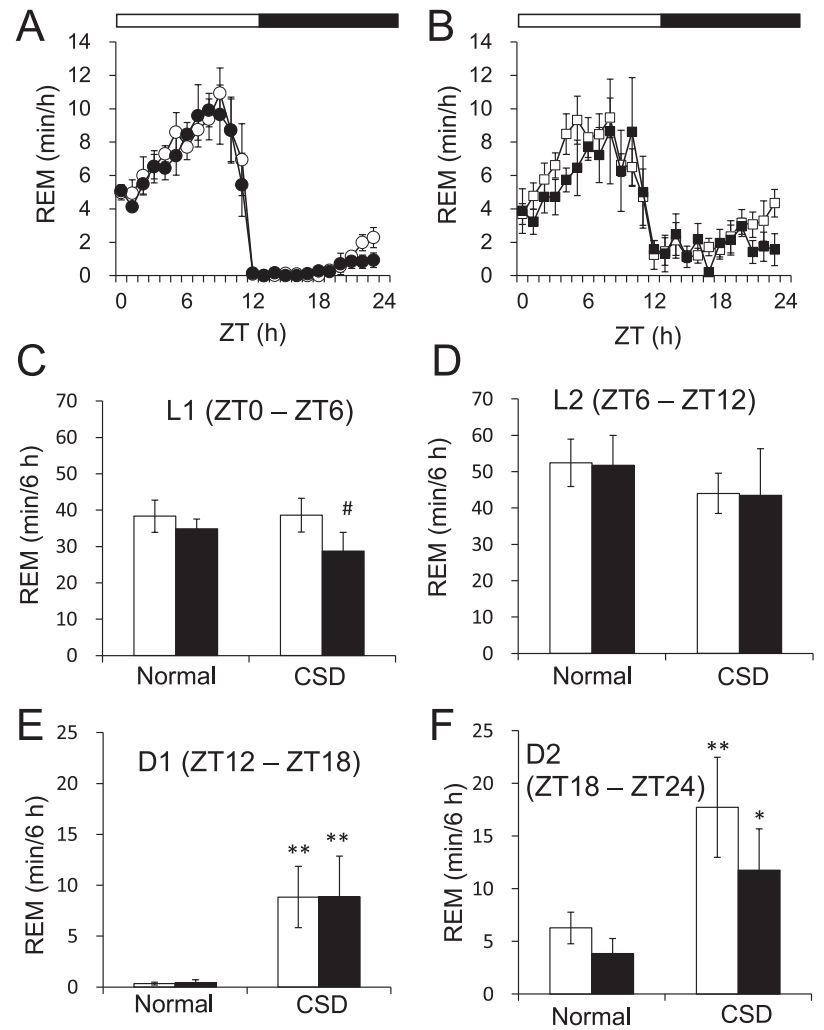

Fig. 5. Dietary heat-killed SBC8803 reduced REM sleep during inactive phase under CSD. Diurnal changes in REM sleep among mice fed with (filled circles) or without (unfilled circles) SBC8803 under normal (A) and CSD (B) conditions. C-F: Total time spent in REM sleep every $6 \mathrm{~h}$ by mice fed with (filled bar) or without (unfilled bar) SBC8803 under normal and CSD conditions. C, ZTO-6 (L1); D, ZT6-12 (L2); E, ZT12-18 (D1); F, ZT18-24 (D2). Data are shown as means \pm SE (Control, $n=5$; SBC8803, $n=4$ ). Significant differences between normal and CSD conditions in each group $\left({ }^{*} p<0.05\right.$ and $\left.{ }^{* *} p<0.01\right)$ and between control and SBC8803 mice under each condition ( $\left.{ }^{\#} p<0.05\right)$. Horizontal unfilled and filled bars indicate light and dark period, respectively.

SBC8803 did not change the power density spectrum in the range of $0.5-20 \mathrm{~Hz}$ compared with control mice (Fig. 7A).

\section{DISCUSSION}

The present study aimed to determine whether SBC8803 improves sleep disruption induced by psychophysiological stress in a mouse model characterized by disrupted circadian rhythms of wheel-running activity and sleep-wake cycles. Dietary SBC8803 suppressed the CSD-induced reduction of wheel-running activity during the active phase, significantly restored wakefulness and increased the duration of NREM sleep during the inactive phase. SBC8803 supplementation significantly improved the CSD-induced reduction of EEG slow wave activity, a marker of NREM sleep intensity, during the beginning of the inactive phase.

Stress can be defined as a disruption in homeosta- 


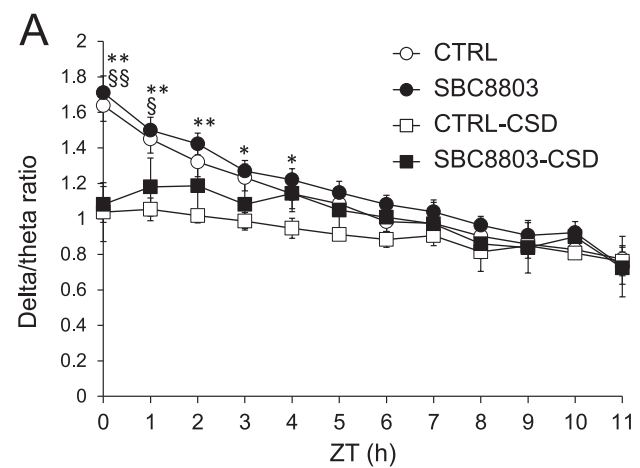

B

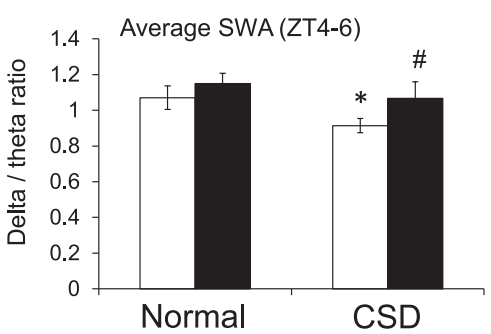

Fig. 6. Dietary heat-killed SBC8803 suppressed the CSD-induced reduction of delta/theta ratio in NREM sleep. A: Hourly time course changes in EEG delta/theta ratios during NREM sleep in mice fed with (filled) or without (unfilled) SBC8803 under normal (circles) and CSD (squares) conditions. B: Average value of EEG delta/ theta ratios in NREM sleep during ZT4-6 among mice fed with (filled bar) or without (unfilled bar) SBC8803 under normal and CSD conditions. Data are shown as means \pm SE (Control, $n=5$; SBC8803, $n=4$ ). Significant differences between normal and CSD conditions in control (CTRL) group $\left({ }^{*} p<0.05\right.$ and $\left.{ }^{* *} p<0.01\right)$, between normal and CSD conditions in SBC8803 group $\left({ }^{\S}<<0.05\right.$ and $\left.\S p<0.01\right)$. and between control and SBC8803 mice under CSD $\left({ }^{\#} p<0.05\right)$.

sis due to environmental, physical or psychological stimuli that elicit adaptive physiological and behavioral responses to restore homeostasis (17). Stress modulates the structure and activity of the gut microbiota, which might be one cause of dysbiosis $(18,19)$. Stress induces the activation of gastrointestinal 5-HT3 receptors via 5-HT released from enterochromaffin cells and nerve terminals (20). Furthermore, 5-HT might be associated with gut-brain function in irritable bowel syndrome, because 5-HT3 antagonists, 5-HT4 agonists, and antidepressants regulate 5-HT neurotransmission in patients with irritable bowel syndrome (21). Receptors for 5-HT3 are widely distributed in the gut, where they are involved in the central and enteric nervous systems (22). These findings suggest that the activation of gastrointestinal 5-HT3 receptors plays important roles in protecting organisms against various stressors. We previously showed that the intraduodenal administration of heat-killed SBC8803 elevates both efferent gastric (efferent GVNA) and afferent intestinal (afferent IVNA) vagal nerve activity in rats (23). Increases in both of efferent GVNA and afferent IVNA due to SBC8803 are abolished by intravenously administered 5-HT3 receptor antagonist (24). Intestinal SBC8803 signals are carried

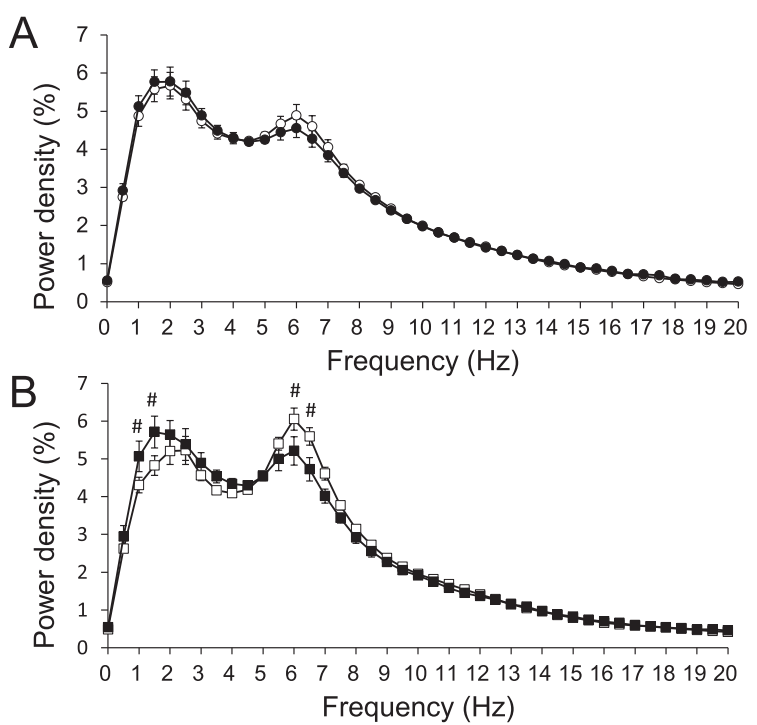

C

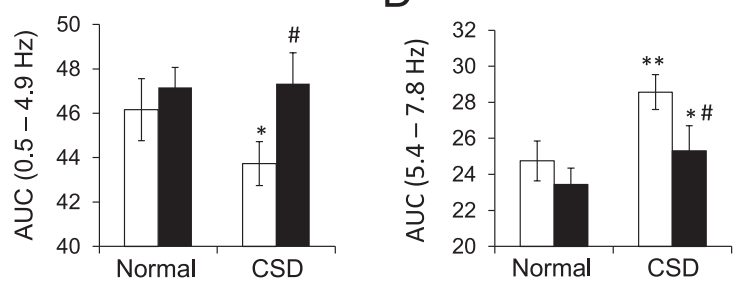

Fig. 7. Dietary heat-killed SBC8803 increased delta wave and decreased theta wave at ZT4 under CSD condition. Spectral analysis of EEG power density (expressed as ratios (\%) of total power spectrum density with frequency ranging from 0 to $19.5 \mathrm{~Hz}$ ) during NREM sleep at ZT4 among mice fed with (filled circles/ squares) or without (unfilled circles/squares) SBC8803 under normal (A) and CSD (B) conditions. Area under the curve (AUC) of EEG power density (\%) in delta frequency band (C: $0.5-4.9 \mathrm{~Hz})$ and theta frequency band (D: $5.4-7.8 \mathrm{~Hz}$ ) are shown. Data are shown as means \pm SE (Control, $n=5$; SBC8803, $n=4$ ). Significant differences between normal and CSD conditions in each group $\left({ }^{*} p<0.05\right.$ and $\left.{ }^{* *} p<0.01\right)$ and between control and SBC8803 mice at each frequency band or under each condition $\left({ }^{\#} p<0.05\right)$.

by afferent vagal nerves to the central nervous system, then disseminated to peripheral organs via efferent vagal nerves (24). SBC8803 also stimulates 5-HT release from cells in the intestinal tract (16). These findings suggest that orally administered SBC8803 prevents psychophysiological stress-induced CSD by affecting gut-brain interactions via gastrointestinal 5-HT3 activation.

The underlying mechanism of psychophysiological stress-induced CSD in the present study is unclear, but the stress applied herein might induce psychological disruptions such as anxiety and depression that are associated with functionally impaired $\gamma$-aminobutyric acid (GABA)-ergic synaptic transmission. Bravo et al. (25) showed that chronic ingestion of Lactobacillus rhamnosus affected region-dependent GABA receptor expression and reduced anxious and depressive behaviors induced by stress in mice. Moreover, these behavioral effects are 
absent in vagotomized mice, suggesting that the vagus is a major modulatory constitutive communication pathway between the brain and bacteria in the gut. As noted above, SBC8803 activates both efferent GVNA and afferent IVNA (23), suggesting that SBC8803 improves CSD by modulating the central expression of GABA receptors.

We previously assessed the effects of SBC8803 consumption in a non-randomized, placebo-controlled, double-blind, crossover pilot study of volunteers with sleep challenges (such as poor-quality sleep, waking during sleep) (11). A statistically significant $(p=0.047)$ effect of SBC8803 consumption was found in the "waking” item of sleep journals. Sleep EEG analysis revealed an increasing trend in the delta power values that was marginally significant $(p=0.065)$ during the period of SBC8803 consumption by individuals with lower-thanaverage Athens Insomnia Scale (AIS) scores and a statistically significant $(p=0.045)$ effect was found in the delta power values among those with lower-than-average Beck Depression Inventory (BDI) scores. The delta power value of five individuals with lower than average BDI and AIS scores significantly increased $(p=0.017)$. These results indicate that persons with considerable sleep challenges are unlikely to benefit from the consumption of heat-killed SBC8803, whereas it appears to show promise for those with sleep challenges that are slight. Our more recent additional randomized, doubleblind, parallel-group, placebo-controlled study investigated sleep quality among healthy Japanese persons who scored their quality of sleep dissatisfaction after $4 \mathrm{wk}$ of SBC8803 intake (26). The per protocol set comprised 103 individuals (sleep test data from all of them) in the intervention group and 101 (sleep test data from 99 of them) in the placebo group. Outcomes were evaluated using the Ogri-Shirakawa-Azumi sleep inventory MA version (OSA-MA) (27) and a sleep scanning mat. The primary outcomes for factors I (sleepiness on rising) and IV (refreshed) in the OSA-MA significantly improved in the intervention group $(p<0.05)$. Sleep efficiency and the occurrence of REM sleep significantly increased, and the appearance of awakening significantly decreased $(p<0.05)$. These findings resembled those of the mice in the present study. SBC8803 consumption suppressed stress-induced sleep disorders such as unusual sleep-wake rhythms and decreased delta power but did not affect mice under normal conditions. On the other hand, we previously showed that heat-killed SBC8803 enhanced running wheel activity and reduced the total amount of time spent in NREM sleep during the latter half of the nighttime under normal conditions (10). Dietary SBC8803 might affect the regulation of circadian behavioral activity and sleep-wake cycles more effectively under unusual conditions.

The present findings suggested that dietary heat-killed SBC8803 can help individuals with insomnia induced by psychophysiological stress and those with circadian sleep disorders. Kim and Dimsdale (28) noted in a systematic review that slow-wave sleep can be reduced by ordinary everyday as well as experimental psychologi- cal stressors. The findings of the CSD mice in the present study supports this notion. The CSD mice seemed to be a useful model for not only studying the underlying mechanisms of stress-induced sleep disorders but also for screening food components that could improve sleep. The components of SBC8803 that can help to regulate sleep in relation to gastrointestinal 5-HT action in CSD mice remain to be elucidated.

\section{Acknowledgments}

This study was supported by the Japan Society for the Promotion of Science (JSPS) KAKENHI Grant Number JP16K00940 (to K. Oishi). We thank Nanako Itoh and Sumika Ohyama (AIST) for technical assistance.

\section{Supporting Information}

Supplemental online material is available on J-STAGE.

\section{REFERENCES}

1) Knutson KL, Spiegel K, Penev P, Van Cauter E. 2007. The metabolic consequences of sleep deprivation. Sleep Med Rev 11: 163-178.

2) Uehli K, Mehta AJ, Miedinger D, Hug K, Schindler C, Holsboer-Trachsler E, Leuppi JD, Künzli N. 2014. Sleep problems and work injuries: a systematic review and meta-analysis. Sleep Med Rev 18: 61-73.

3) Kalmbach DA, Anderson JR, Drake CL. 2018. The impact of stress on sleep: Pathogenic sleep reactivity as a vulnerability to insomnia and circadian disorders. J Sleep Res 27: e12710.

4) Szajewska H, Mrukowicz JZ. 2001. Probiotics in the treatment and prevention of acute infectious diarrhea in infants and children: a systematic review of published randomized, double-blind, placebo-controlled trials. J Pediatr Gastroenterol Nutr 33(Suppl 2): S17-25.

5) Moayyedi P, Ford AC, Talley NJ, Cremonini F, Foxx-Orenstein AE, Brandt LJ, Quigley EM. 2010. The efficacy of probiotics in the treatment of irritable bowel syndrome: a systematic review. Gut 59: 325-332.

6) Segawa S, Nakakita Y, Takata Y, Wakita Y, Kaneko T, Kaneda H, Watari J, Yasui H. 2008. Effect of oral administration of heat-killed Lactobacillus brevis SBC8803 on total and ovalbumin-specific immunoglobulin E production through the improvement of Th1/Th2 balance. Int J Food Microbiol 121: 1-10.

7) Ueno N, Fujiya M, Segawa S, Nata T, Moriichi K, Tanabe H, Mizukami Y, Kobayashi N, Ito K, Kohgo Y. 2011. Heat-killed body of Lactobacillus brevis SBC8803 ameliorates intestinal injury in a murine model of colitis by enhancing the intestinal barrier function. Inflamm Bowel Dis 17: 2235-2250.

8) Segawa S, Wakita Y, Hirata H, Watari J. 2008. Oral administration of heat-killed Lactobacillus brevis SBC8803 ameliorates alcoholic liver disease in ethanolcontaining diet-fed C57BL/6N mice. Int J Food Microbiol 128: $371-377$.

9) Wakita Y, Kanda H, Shimizu C, Nakakita Y, Kaneda H, Segawa S, Ozaki M, Shigyo T, Ohtake T, Fujiya M, Kohgo Y. 2012. Effect of Lactobacillus brevis SBC8803 on gamma-glutamyl transferase in Japanese habitual drinkers: A double-blind, placebo-controlled study. Food Nutr Sci 3: 678-684.

10) Miyazaki K, Itoh N, Yamamoto S, Higo-Yamamoto S, Nakakita Y, Kaneda H, Shigyo T, Oishi K. 2014. Dietary 
heat-killed Lactobacillus brevis SBC8803 promotes voluntary wheel-running and affects sleep rhythms in mice. Life Sci 111: 47-52.

11) Nakakita $Y$, Tsuchimoto $N$, Takata $Y$, Nakamura $T$. 2016. Effect of dietary heat-killed Lactobacillus brevis SBC8803 (SBL88) on sleep: a non-randomised, double blind, placebo-controlled, and crossover pilot study. Benef Microbes 7: 501-509.

12) Toth LA, Bhargava P. 2013. Animal models of sleep disorders. Comp Med 63: 91-104.

13) Miyazaki K, Itoh N, Ohyama S, Kadota K, Oishi K. 2013. Continuous exposure to a novel stressor based on water aversion induces abnormal circadian locomotor rhythms and sleep-wake cycles in mice. PLoS One 8: e 55452.

14) Oishi K, Ohyama S, Higo-Yamamoto S. 2018. Chronic sleep disorder induced by psychophysiological stress induces glucose intolerance without adipose inflammation in mice. Biochem Biophys Res Commun 495: 2616-2621.

15) Oishi K, Yamamoto S, Itoh N, Miyazaki K, Nemoto T, Nakakita Y, Kaneda H. 2014. Disruption of behavioral circadian rhythms induced by psychophysiological stress affects plasma free amino acid profiles without affecting peripheral clock gene expression in mice. Biochem Biophys Res Commun 450: 880-884.

16) Nakaita Y, Kaneda H, Shigyo T. 2013. Heat-killed Lactobacillus brevis SBC8803 induces serotonin release from intestinal cells. Food Nutr Sci 4: 767-771.

17) Mackos AR, Maltz R, Bailey MT. 2017. The role of the commensal microbiota in adaptive and maladaptive stressor-induced immunomodulation. Horm Behav 88: 70-78.

18) Sudo N, Chida Y, Aiba Y, Sonoda J, Oyama N, Yu XN, Kubo C, Koga Y. 2004. Postnatal microbial colonization programs the hypothalamic-pituitary-adrenal system for stress response in mice. J Physiol 558(Pt 1): 263-275.

19) Karl JP, Hatch AM, Arcidiacono SM, Pearce SC, PantojaFeliciano IG, Doherty LA, Soares JW. 2018. Effects of psychological, environmental and physical stressors on the gut microbiota. Front Microbiol 9: 2013.

20) Miyata K, Kamato T, Nishida A, Ito H, Yuki H, Yamano M, Tsutsumi R, Katsuyama Y, Honda K. 1992. Role of the serotonin 3 receptor in stress-induced defecation. J Pharmacol Exp Ther 261: 297-303.

21) Fukudo S. 2013. Stress and visceral pain: focusing on irritable bowel syndrome. Pain 154(Suppl 1): S63-70.

22) Hirata T, Keto Y, Nakata M, Takeuchi A, Funatsu T, Akuzawa S, Sasamata M, Miyata K. 2008. Effects of serotonin 5-HT3 receptor antagonists on stress-induced colonic hyperalgesia and diarrhoea in rats: a comparative study with opioid receptor agonists, a muscarinic receptor antagonist and a synthetic polymer. Neurogastroenterol Motil 20: 557-565.

23) Horii Y, Nakakita Y, Fujisaki Y, Yamamoto S, Itoh N, Miyazaki K, Kaneda H, Oishi K, Shigyo T, Nagai K. 2013. Effects of intraduodenal injection of Lactobacillus brevis SBC8803 on autonomic neurotransmission and appetite in rodents. Neurosci Lett 539: 32-37.

24) Horii Y, Nakakita Y, Fujisaki Y, Kaneda H, Shigyo T, Nagai K. 2015. Intraduodenal injection of heat-killed Lactobacillus brevis SBC8803 modulates autonomic neurotransmission in the gut: involvement of vagal afferents and serotonin receptors. Curr Topics Nutraceutical Res 13: 47-54.

25) Bravo JA, Forsythe P, Chew MV, Escaravage E, Savignac HM, Dinan TG, Bienenstock J, Cryan JF. 2011. Ingestion of Lactobacillus strain regulates emotional behavior and central GABA receptor expression in a mouse via the vagus nerve. Proc Natl Acad Sci USA 108: 16050-16055.

26) Yoshikawa K, Aota K, Takata Y, Nakamura T, Hoshino T, Yamashita S, Takara T. 2018. Efficacy of the Lactobacillus brevis SBC8803 (SBL88 ${ }^{\mathrm{TM}}$ ) intake on the quality of sleep: A randomized, double-blind, parallel-group, placebo-controlled study. Jpn Pharmacol Ther 46: 17231738 (in Japanese).

27) Miyake M, Kirisako T, Kokubo T, Miura Y, Morishita K, Okamura H, Tsuda A. 2014. Randomised controlled trial of the effects of L-ornithine on stress markers and sleep quality in healthy workers. Nutr J 13: 53.

28) Kim EJ, Dimsdale JE. 2007. The effect of psychosocial stress on sleep: a review of polysomnographic evidence. Behav Sleep Med 5: 256-278. 